\title{
Studio EVOLVE: un'altra delusione per i Nefrologi?
}

\author{
Andrea Cavalli' ${ }^{1}$, Giuseppe Pontoriero ${ }^{2}$ \\ ${ }^{1}$ S.C. Nefrologia e Dialisi, Ospedale Civile di Sondrio, Sondrio \\ ${ }^{2}$ S.C. Nefrologia e Dialisi, Ospedale “Alessandro Manzoni”, Lecco
}

THE EVOLVE STUDY: ANOTHER DISAPPOINTMENT FOR NEPHROLOGISTS?

Abstract. The calcimimetic cinacalcet is now being used for years to treat secondary hyperparathyroidism (SHPT) in dialysis patients, allowing a good reduction in PTH levels. In order to evaluate a possible beneficial effect on cardiovascular prognosis, a randomized trial, the EVOLVE (Evaluation of Cinacalcet Hydrochloride Therapy to Lower Cardiovascular Events), was conducted in 3,883 dialysis patients affected by moderate to severe SHPT.

Patients were randomly assigned to receive cinacalcet or placebo. The primary composite endpoint evaluated was time until death, myocardial infarction, hospitalization for unstable angina, heart failure or peripheral vascular events. Due to a low statistical power, there was no significant difference between the two arms of the study in the intention-to-treat analysis, despite a higher incidence of hypocalcaemia, nausea, and vomiting in patients treated with cinacalcet.

The results of the EVOLVE study have certainly disappointed the nephrological community that would have expected a positive conclusion. Lack of conclusive results and high costs suggest a judicious use of cinecalcet. Nevertheless, even in the absence of conclusive data on hard endpoints it would be unwise to deny the availability of therapeutic strategies that include cinacalcet to patients with severe SHPT and high levels of PTH (PTH $>800 \mathrm{pg} / \mathrm{mL})$ refractory to standard therapy and/or hypercalcaemia and/or calciphylaxis and/or high surgical risk to parathyroidectomy.

Key words: Cinacalcet, Calcimimetic, Secondary hyperparathyroidism, Chronic kidney disease, Mortality, Cardiovascular events

Conflict of interest: None.

Ricevuto: 12 Agosto 2013; Accettato: 1 Ottobre 2013

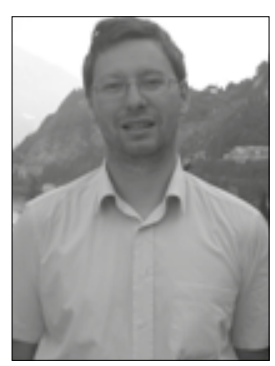

Andrea Cavalli
Era la primavera dell'ormai lontano 2004 quando, sull'autorevole New England Journal of Medicine venivano pubblicati da Geoffrey Block et al. i risultati di un trial randomizzato mondiale condotto in oltre 700 pazienti emodializzati affetti da iperparatiroidismo secondario (IPTS) non adeguatamente controllato, in cui la somministrazione del calciomimetico Cinacalcet si dimostrava in grado di ridurre significativamente i livelli di paratormone (PTH) (1).

Da allora, il Cinacalcet è entrato nell'armamentario terapeutico nefrologico per il trattamento dell'IPTS nei pazienti dializzati, per la riduzione dell'ipercalcemia in caso di carcinoma paratiroideo e iperparatiroidismo primario (2) e nei soggetti portatori di trapianto renale con iperparatiroidismo secondario (3) (Tab. I).

Nell'articolo di Block (1) veniva sottolineato come la più comune complicanza dell'IPTS fosse la malattia ossea (4), suggerendo, però, come lo stesso IPTS potesse anche contribuire alla comparsa di calcificazioni vascolari e dei tessuti molli e di malattie cardio-vascolari e alla riduzione della sopravvivenza dei pazienti affetti da malattia renale cronica (IRC), tanto da essere annoverato, insieme a iperfosforemia e a ipercalcemia, tra i fattori di rischio cardio-vascolare tipicamente associati all'IRC (5). Anche alla luce dei risultati di due studi successivi, condotti utilizzando il Cinacalcet, che mostravano una riduzione di eventi cardio-vascolari, fratture, paratiroidectomie (6) e progressione delle calcificazioni vascolari e valvolari cardiache (7), è stato disegnato il trial "Evaluation of Cinacalcet Hydrochloride Therapy to Lower Cardiovascular Events" (EVOLVE) (8), come, peraltro, auspicato anche nello studio di Block (1), per verificare se il Cinacalcet potesse ridurre il rischio di morte e di eventi cardio-vascolari non fatali.

Lo studio EVOLVE, multicentrico, prospettico, randomizzato, in doppio cieco e controllato, ha confrontato l'effetto del Cinacalcet, in aggiunta alla terapia standard (chelanti del fosforo e supplementi di calcio e vitamina $\mathrm{D}$, in accordo con quanto suggerito dalle Linee Guida), rispetto al placebo in un totale di 3883 pazienti emodializzati (1948 vs 1935 rispettivamente), affetti da IPTS moderato-severo (PTH mediano di $693 \mathrm{pg} / \mathrm{mL}, 10^{\circ}$ per- 
TABELLA I - ATTUALI INDICAZIONI ALL'UTILIZZO DEL CINACALCET

\footnotetext{
1. Iperparatiroidismo secondario in pazienti dializzati

2. Riduzione dell'ipercalcemia in pazienti con carcinoma paratiroideo

3. Riduzione dell'ipercalcemia in pazienti con iperparatiroidismo primario (nei quali la paratiroidectomia non è clinicamente appropriata $\mathrm{o}$ è controindicata)

4. Riduzione dell'ipercalcemia indotta da iperparatiroidismo secondario in pazienti portatori di trapianto renale
}

centile pari a $363 \mathrm{pg} / \mathrm{mL}$ e $90^{\circ}$ percentile pari a 1694) arruolati tra l'Agosto del 2006 e il Gennaio del 2008 (9). Al basale, non c'erano differenze significative tra i due gruppi, a eccezione della pressione diastolica media e degli attacchi ischemici transitori. L'età mediana dei pazienti era relativamente bassa $\mathrm{e}$, tra $\mathrm{i}$ due gruppi, c'era uno squilibrio di 1 anno (Cinacalcet 55 anni, placebo 54 anni). Purtroppo, la randomizzazione non prevedeva una stratificazione per questo fondamentale fattore prognostico di morte ed eventi cardio-vascolari.

L'end-point composito primario era rappresentato dalla morte o dal primo evento cardio-vascolare non fatale (infarto miocardico, angina instabile richiedente l'ospedalizzazione, scompenso cardiaco, evento vascolare periferico), mentre gli end-point secondari includevano i singoli componenti dell'end-point primario, la morte per cause cardio-vascolari, gli episodi di ictus, le fratture ossee e il ricorso all'intervento di paratiroidectomia (8). Il follow-up dei pazienti è durato fino a 64 mesi, con un'esposizione mediana al Cinacalcet pari a 21.2 mesi e al placebo di 17.5 mesi e una dose mediana di $55 \mathrm{mg}$ e $125 \mathrm{mg} /$ die, rispettivamente. Occorre sottolineare che, nel gruppo randomizzato al calciomimetico, ben il $62 \%$ dei pazienti (1300) ha interrotto l'assunzione del farmaco in studio, soprattutto per ragioni non pre-specificate dal protocollo (562), eventi avversi (308) e bassi livelli di PTH (101). Nel gruppo placebo, invece, quasi il $20 \%$ dei pazienti ha iniziato l'assunzione di "Cinacalcet commerciale" prima dell'insorgenza di un end-point (10).

Nell'analisi "intention-to-treat" non aggiustata, come previsto nel disegno dello studio, l'end-point composito primario si è verificato in 938 (48.2\%) e 952 (49.2\%) soggetti nei gruppi Cinacalcet e placebo rispettivamente, determinando una differenza statisticamente non significativa tra i due bracci di trattamento del 7\% (rischio relativo (HR) Cinacalcet vs placebo 0.93 ; intervallo di confidenza al $95 \%$ (95\% IC): 0.85-1.02; $\mathrm{P}=0.11$ ), che diveniva, però, rilevante, e pari a una riduzione del rischio del $12 \%$, dopo aver corretto i risultati per le caratteristiche di base dei due gruppi (HR 0.88; 95\% IC, 0.79-0.97; $\mathrm{P}=0.008$ ). L'effetto del Cinacalcet era più pronunciato nei pazienti con età $>65$ anni ( $\mathrm{P}=0.03$ per l'interazione) (10). Bisogna sottolineare che, se l'end-point primario è statisticamente non significativo, non si può procedere a testare formalmente la significatività statistica degli end-point secondari. Nel nostro caso, questo significa che, essendo l'end-point primario non significativo, gli altri valori di P sono "nominali", cioè i valori di P sono descrittivi e formalmente non si può rivendicarne una significatività statistica.

È stata condotta un'analisi di accompagnamento, pre-specificata, censurando i dati dei pazienti dopo sei mesi dalla sospensione del farmaco in studio. Con una simile analisi si otteneva una riduzione nominalmente significativa dell'end-point primario nel gruppo del Cinacalcet rispetto al gruppo placebo (HR 0.85; 95\% IC, 0.76-0.96; $\mathrm{P}=0.009)$. Analogamente, censurando i dati dei pazienti dopo il trapianto, la paratiroidectomia e il passaggio all'assunzione di "Cinacalcet commerciale", il rischio relativo per l'end-point primario risultava nominalmente significativo e pari a $0.90(95 \%$ IC, $0.82-0.99$; $\mathrm{P}=0.03)(10)$. Non sono emerse differenze tra i due gruppi analizzando i singoli componenti dell'end-point primario, a parte una riduzione degli episodi di scompenso cardiaco in corso di calciomimetico (HR 0.82; $95 \%$ IC, 0.68-0.99; $\mathrm{P}=0.03)(10)$.

Per quanto riguarda gli altri end-point secondari, merita di essere segnalata l'incidenza della paratiroidectomia, eseguita in 140 (7\%) e 278 (14\%) soggetti nel braccio Cinacalcet e placebo, rispettivamente, permettendone, quindi, una riduzione del $50 \%$ (HR 0.44; 95\% IC, 0.36-0.54; $\mathrm{P}<0.001$ ). Invece, non sono state rilevate differenze tra i due gruppi nell'incidenza di fratture (12\% Cinacalcet vs $13 \%$ placebo) (10).

Tuttavia, il risultato forse più eclatante dello studio riguardava l'elevata incidenza di eventi avversi nel braccio sperimentale: $45.9 \%$ dei soggetti rispetto al $18.9 \%$ nel braccio di controllo. Nello specifico, i pazienti che ricevevano il calciomimetico sviluppavano ipocalcemia (intesa come calcemia $<8.0 \mathrm{mg} / \mathrm{dL} / 2.00$ $\mathrm{mmol} / \mathrm{L}$ ) sette volte più frequentemente rispetto ai pazienti del gruppo placebo ( $12.4 \%$ vs $1.7 \%$ ), oltre a presentare nausea e vomito con un'incidenza doppia (rispettivamente $29.1 \%$ vs $15.5 \%$ e $25.6 \%$ vs $13.7 \%$ ). Pertanto, il $18.1 \%$ dei soggetti randomizzati a Cinacalcet (rispetto al 13\% dei soggetti randomizzati a placebo) ha sospeso la terapia a causa della comparsa di effetti collaterali. Occorre sottolineare, però, che l'incidenza di eventi avversi seri era sovrapponibile (10).

Purtroppo, per noi nefrologi, i risultati di questo trial vanno ad aggiungersi ad analoghi esiti emersi in precedenti studi disegnati per valutare diversi interventi che potessero migliorare la prognosi generale e cardio-vascolare dei pazienti dializzati: l'inizio precoce della terapia sostitutiva (11), l'aumento della dose di dialisi (12), la normalizzazione dell'ematocrito (13) e la terapia con statine (14-16), per citarne solo alcuni.

Gli Autori dello studio EVOLVE sottolineano comunque come la riduzione del rischio, non significativa, del $7 \%$ di end-point primario nell'analisi "intention-to-treat" diventi poi, però, nominalmente significativa, e pari al $12 \%$, dopo aver "aggiustato i dati" in accordo con le caratteristiche di base dei soggetti arruolati, sottintendendo che il trial avrebbe potuto avere tutt'altro risultato se la randomizzazione avesse funzionato più appropriatamente. Inoltre, va riconosciuto come gli interventi di paratiroidectomia e l'inizio della terapia con "Cinacalcet commerciale" abbiano sicuramente ridotto i livelli di PTH e le differenze tra i due gruppi, teoricamente spostando l'effetto del trattamento sperimentale verso risultati negativi.

Punti di forza dello studio sono la sua "multicentricità", la possibile concomitante terapia con chelanti del fosforo e vitamina $\mathrm{D}$ e la rigorosa e indipendente assegnazione degli end-point cardio-vascolari. Tuttavia, gli stessi Autori riconoscono al trial importanti limiti. Lo studio EVOLVE era stato disegnato per avere una potenza statistica sufficiente per mettere in evidenza una riduzione di rischio del $20 \%$ per l'end-point primario 
nel gruppo trattato con Cinacalcet. Lo studio era programmato per concludersi quando si fosse verificato un numero di eventi primari sufficiente per ottenere la desiderata potenza statistica del $90 \%$. Per ottenere questo risultato, era stato calcolato che si dovesse realizzare un end-point primario in 1882 pazienti, presupponendo un tasso di eventi primari del $23.2 \%$ nel gruppo placebo, un drop-out (sospensione del farmaco in studio prima che si realizzasse un evento primario) del 10\% nel gruppo del Cinacalcet e un drop-in (assunzione del Cinacalcet commerciale prima di qualsiasi evento primario) del $10 \%$ nel gruppo placebo. In effetti, il tasso di eventi primari è stato più basso dell'atteso e il drop-out molto superiore rispetto a quanto preventivato. È stato, quindi, necessario prolungare lo studio di altri 16 mesi, oltre i 4 anni preventivati. Questo prolungamento, a sua volta, ha magnificato l'effetto distorsivo dell'eccessivo drop-out/drop-in. Gli Autori hanno stimato che la potenza statistica dello studio è pari al 54\%, se si tiene conto di dati reali di durata dello studio, tasso dell'evento primario, drop-out e drop-in e se si mantiene l'assunzione iniziale di una riduzione del rischio del $20 \%$ nel gruppo del Cinacalcet (10).

Numerose lettere di commento sono giunte al New England Journal of Medicine, volte a sottolineare gli elementi di criticità dello studio EVOLVE. Tra queste, Pablo Urena (17) ritiene che i risultati negativi del trial siano da imputare a un'inadeguata definizione di IPTS, indicata unicamente da livelli di PTH superiori a $300 \mathrm{pg} / \mathrm{mL}$ (erano, pertanto, arruolabili anche pazienti con IPTS moderato), senza riferimento a parametri di turn-over osseo. Analogamente, è stato messo in discussione il reale significato del PTH come marcatore di prognosi generale e cardiovascolare, così come opinabile sarebbe il target, probabilmente troppo "stretto", di PTH desiderato (150-300 pg/mL), anche alla luce delle odierne Linee Guida (18). Francesco Locatelli, invece, segnala quanto, da un punto di vista metodologico e statistico, sia spesso difficile attenersi unicamente a "un'analisi basata sull'intention-to-treat", soprattutto quando si devono prendere in considerazione i vantaggi e gli svantaggi per il singolo paziente conseguenti all'assunzione di un farmaco (19). I limiti e le criticità dello studio EVOLVE sono riassunti nella Tabella II. Palmer et al. hanno recentemente pubblicato i risultati di una meta-analisi che ha preso in considerazione 18 studi randomizzati e controllati che hanno valutato gli effetti del Cinacalcet su mortalità ed eventi avversi in pazienti con malattia renale cronica (per un totale di 7446 soggetti). Nei pazienti dializzati è emerso come il Cinacalcet, utilizzato come terapia di "routine" o di "prima linea", non determini effetti rilevanti su mortalità generale e cardio-vascolare, ma sia in grado di prevenire la paratiroidectomia e l'ipercalcemia, a costo, però, di un netto incremento degli episodi di ipocalcemia, nausea e vomito. Nello specifico, con una simile strategia, trattando 1000 dializzati per un anno non si otterranno benefici in termini di sopravvivenza, si eviteranno 3 paratiroidectomie, ma 60 soggetti presenteranno ipocalcemia e ben 150 nausea (20). Palmer sottolinea come, nonostante l'incertezza dei benefici, i costi del Cinacalcet siano progressivamente aumentati nel corso degli anni e come il Cinacalcet, negli USA, rappresenti il farmaco più costoso assunto dai dializzati, oltre che una considerevole percentuale della spesa sanitaria in più nazioni (20).

È d'obbligo concludere la nostra rubrica con l'analisi dell'edito- riale di accompagnamento allo studio EVOLVE, che lancia una serie di provocazioni riguardanti le difficoltà che si incontrano nel condurre trial con un lungo follow-up e in "pazienti complicati", l'utilità del Cinacalcet e la necessità di modificare l'attuale processo regolatorio di sviluppo dei farmaci (21). L'elevata incidenza di crossover tra i due bracci di trattamento e lo sbilancio di età al basale sono interpretati come le principali spiegazioni dei risultati negativi dello studio che avrebbero potuto essere, altrimenti, positivi. Gli Autori sottolineano che, al di là dei risultati, l'esperienza dello studio EVOLVE pone due problemi di ordine generale: 1) l'inadeguatezza del processo regolatorio che consente di registrare e utilizzare in modo esteso farmaci per cui non ci sono forti evidenze di efficacia; 2) la propensione della comunità clinica nefrologica a prescrivere farmaci per cui non esistono ancora prove definitive di efficacia, cosa che si è confermata anche nell'ambito di un trial controllato come l'EVOLVE, contribuendo a ridurne la qualità dei risultati.

Se molti ritengono deludenti i risultati $(17,18,20,21)$, gli Autori dell'EVOLVE considerano il loro trial un "trionfo" (22). Dal nostro punto di vista, i risultati dello studio sono semplicemente non conclusivi, perché la potenza statistica non era sufficiente per verificare l'ipotesi testata e, nonostante la randomizzazione, la situazione basale era sorprendentemente sbilanciata per l'età. L'età media dei pazienti dello studio (54.4 \pm 14.4 anni) era, peraltro, piuttosto bassa (9), e ciò genera perplessità sull'applicabilità dei risultati dello studio in pazienti come i nostri dializzati, che, generalmente, sono molto più anziani. In questo caso, tuttavia, va tenuto conto che proprio l'età avanzata ( $>65$ anni) sembra essere un fattore che migliora i risultati della terapia con Cinacalcet $(10,22,23)$.

La mancanza di risultati definitivi e i costi elevati indicano la necessità di un uso giudizioso del Cinacalcet. Non di meno, anche se mancano dati definitivi e anche se verosimilmente non verranno effettuati altri studi controllati sull'effetto del Cinacalcet su "hard end-point" in dialisi, ci sembra poco saggio risparmiare sulla possibilità di strategie terapeutiche che comprendano il Ci-

\section{TABELLA II - LIMITI E CRITICITÀ DELLO STUDIO EVOLVE}

1. Età media (54.4 \pm 14.4 anni) relativamente bassa rispetto ai tipici pazienti dell'emodialisi

2. Differenza, al basale, di un anno di età tra i due gruppi di trattamento a confronto

3. Frequenza di eventi primari inferiore al previsto

4. Prolungamento della durata dello studio: 16 mesi in più rispetto al previsto

5. Elevato dropout nel gruppo sperimentale: sospensione del cinacalcet nel $62 \%$ dei pazienti

6. Elevato drop-in nel gruppo di controllo: utilizzo di "cinacalcet commerciale" in circa il $20 \%$ dei pazienti

7. Differente esposizione tra farmaco in studio (cinacalcet) e placebo

8. Bassa potenza statistica: circa $54 \%$ rispetto al programmato $90 \%$

9. Elevata incidenza di eventi avversi nel braccio randomizzato a cinacalcet 
nacalcet per le classi di pazienti che più potrebbero giovarsene: pazienti con severo IPTS, con elevati livelli di PTH $(>800$ pg/ $\mathrm{mL}$ ), refrattari alla terapia standard e/o con ipercalcemia e/o con calcifilassi e/o con elevato rischio chirurgico alla paratiroidectomia (24).

Per finire, va ricordato che la ricerca farmacologica ha sviluppato nuove molecole di calciomimetici somministrabili per via endovenosa che, oltre a migliorare la compliance farmacologica, promettono di essere tollerati meglio (25). La saga dei calciomimetici non è finita e riserva nuove sorprese.

\section{Riassunto}

Il calciomimetico Cinacalcet è ormai in uso da alcuni anni per il trattamento dell'iperparatiroidismo secondario (IPTS) nei pazienti dializzati, permettendo una buona riduzione dei livelli di paratormone. Nell'ottica di valutare un suo possibile effetto benefico nel migliorare la prognosi cardio-vascolare dei pazienti dializzati, è stato condotto lo studio randomizzato "Evaluation of Cinacalcet Hydrochloride Therapy to Lower Cardiovascular Events" (EVOLVE). Sono stati randomizzati a ricevere Cinacalcet o placebo 3883 pazienti dializzati affetti da iperparatiroidismo secondario moderato-severo, valutando come end-point compositi primari la mortalità e gli episodi di infarto miocardico, l'ospedalizzazione per angina instabile, lo scompenso cardiaco ed eventi vascolari periferici. Nell'analisi "intention-to-treat", a causa di una bassa potenza statistica, non sono state rilevate differenze significative tra i due bracci dello studio, a fronte di una più elevata incidenza di ipocalcemia, nausea e vomito nei soggetti in terapia con il calciomimetico.

I dati dello studio EVOLVE hanno sicuramente deluso la comunità nefrologica, che si sarebbe aspettata dei risultati positivi. La mancanza di risultati conclusivi e i costi elevati suggeriscono un uso giudizioso del Cinacalcet. Non di meno, anche se mancano dati conclusivi su "hard endpoint", ci sembra poco saggio negare strategie terapeutiche che includano il Cinacalcet ai pazienti con severo IPTS con elevati livelli di PTH (PTH $>800 \mathrm{pg} / \mathrm{mL}$ ) refrattari alla terapia standard e/o ipercalcemia e/o calcifilassi e/o elevato rischio chirurgico alla paratiroidectomia.

Parole chiave: Cinacalcet, Calciomimetico, Iperparatiroidismo secondario, Malattia renale cronica, Mortalità, Eventi cardiovascolari

Dichiarazione di conflitto di interessi: Gli Autori dichiarano di non avere conflitto di interessi.

Indirizzo degli Autori:

Dr. Giuseppe Pontoriero

S.C. Nefrologia e Dialisi

Ospedale "Alessandro Manzoni”"

Via Dell'Eremo 9/11

23900 Lecco

g.pontoriero@ospedale.lecco.it

\section{Bibliografia}

1. Block GA, Martin KJ, de Francisco AL, et al. Cinacalcet for secondary hyperparathyroidism in patients receiving hemodialysis. N Engl J Med 2004; 350: 1516-25.

2. Locatelli F, Limardo M, Pontoriero G. New approaches to treatment of secondary hyperparathyroidism. Curr Opin Investig Drugs 2008; 9 (4): 363-70.

3. Cohen JB, Gordon CE, Balk EM, Francis JM. Cinacalcet for the treatment of hyperparathyroidism in kidney transplant recipients: a systematic review and meta-analysis. Transplantation 2012; 94 (10): 1041-8.

4. Hruska KA, Teitelbaum SL. Renal osteodystrophy. N Engl J Med 1995; 333: 166-74.

5. Kidney Disease Improving Global Outcomes (KDIGO) CKDMBD Work Group. KDIGO clinical practice guideline for the diagnosis, evaluation, prevention, and treatment of chronic kidney disease-mineral and bone disorder (CKD-MBD). Kidney Int Suppl 2009; 76: S1-S130.
6. Cunningham J, Danese M, Olson K, Klassen P, Chertow GM. Effects of the calcimimetic cinacalcet $\mathrm{HCl}$ on cardiovascular disease, fracture, and health-related quality of life in secondary hyperparathyroidism. Kidney Int 2005; 68: 1793-800.

7. Raggi P, Chertow GM, Block GA, et al. The ADVANCE study: a randomized trial to evaluate the effects of cinacalcet plus low dose vitamin D on vascular calcification in patients on hemodialysis. Nephrol Dial Transplant 2011; 26: 1327-39.

8. Chertow GM, Pupim LB, Block GA, et al. EVOLVE (Evaluation of Cinacalcet Therapy to Lower Cardiovascular Events): rationale and design of a randomized clinical trial. Clin J Am Soc Nephrol 2007; 2: 898-905.

9. Chertow GM, Correa-Rotter R, Block GA, et al. Baseline characteristics of subjects enrolled in the Evaluation of Cinacalcet $\mathrm{HCl}$ Therapy to Lower Cardiovascular Events (EVOLVE) trial. Nephrol Dial Transplant 2012; 27: 2872-9.

10. Chertow GM, Block GA, Correa-Rotter R, et al. Effect of cinacalcet on cardiovascular disease in patients undergoing dialysis. N Engl J Med 2012; 367 (26): 2482-94. 
11. Cooper BA, Branley P, Bulfone L, et al. A randomized, controlled trial of early versus late initiation of dialysis. N Engl J Med 2010; 363: 609-19.

12. Eknoyan G, Beck GJ, Cheung AK, et al. Effect of dialysis dose and membrane flux in maintenance hemodialysis. N Engl J Med 2002; 347: 2010-9.

13. Besarab A, Bolton WK, Browne JK, et al. The effects of normal as compared with low hematocrit values in patients with cardiac disease who are receiving hemodialysis and epoetin. $\mathrm{N}$ Engl $\mathrm{J}$ Med 1998; 339: 584-90.

14. Wanner C, Krane V, März W, et al. Atorvastatin in patients with type 2 diabetes mellitus undergoing hemodialysis. N Engl J Med 2005; 353: 238-48.

15. Fellström BC, Jardine AG, Schmider RE, et al. Rosuvastatin and cardiovascular events in patients undergoing hemodialysis. N Engl J Med 2009; 360: 1395-407.

16. Baigent $\mathrm{C}$, Landray MJ, Reith $\mathrm{C}$, et al. The effects of lowering LDL cholesterol with simvastatin plus ezetimibe in patients with chronic kidney disease (Study of Heart and Renal Protection): a randomized placebo-controlled trial. Lancet 2011; 377: 2181-92.

17. Urena-Torres P, Prié D, Souberbielle JC. Cinacalcet for cardiovascular disease in patients undergoing dialysis. N Engl J Med 2013; 368 (19): 1842-3.
18. Goldsmith DJ, Lamb EJ. Cinacalcet for cardiovascular disease in patients undergoing dialysis. N Engl J Med 2013; 368 (19): 1843.

19. Locatelli F, Pontoriero G, Tentori F. Cinacalcet for cardiovascular disease in patients undergoing dialysis. N Engl J Med 2013; 368 (19): 1843.

20. Palmer SC, Nistor I, Craig JC, et al. Cinacalcet in patients with chronic kidney disease: a cumulative meta-analysis of randomized controlled trials. PLoS Med 2013; 10 (4): e1001436.

21. Perkovic V, Neal B. Trials in kidney disease--time to EVOLVE. N Engl J Med 2012; 367 (26): 2541-42.

22. Chertow GM, Parfrey PS. Cinacalcet for cardiovascular disease in patients undergoing dialysis. N Engl J Med 2013; 368 (19): 1844-5.

23. Tripepi G. Cinacalcet for cardiovascular disease in patients undergoing dialysis. N Engl J Med 2013; 368 (19): 1843-4.

24. National Institute for Health and Clinical Excellence. Hyperparathyroidism-cinacalcet (TA117). Available: http://www.nice. org.uk/ta117. Accessed July 2013.

25. Martin KJ, Pickthorn K, Huang S, et al. AMG 416 (velcalcetide) is a novel peptide for the treatment of secondary hyperparathyroidism in a single-dose study in hemodialysis patients. Kidney Int 2013. doi: 10.1038/ki.2013.289. 